\title{
New ro-vibrational kinetic energy operators using polyspherical coordinates for polyatomic molecules
}

\author{
David W. Schwenke \\ NASA Ames Research Center, Moffett Field, CA 94035-1000
}

(Dated: Draft version December 19, 2002)

\begin{abstract}
We illustrate how one can easily derive kinetic energy operators for polyatomic molecules using polyspherical coordinates with very general choices for z-axis embecldings and angles used to specify relative orientations of internal vectors. Computer algebra is not required.
\end{abstract}




\section{INTRODUCTION}

Mladenović[1, 2] and Gatti et al. [3 5] have presented derivations of kinetic energy operators for treating rotations and vibrations of polyatomic molecules using polyspherical coordinates. In these coordinates, the $N$ nuclear position vectors are transformed to $N-1$ internal vectors and the nuclear center of mass vector, and the internal vectors are parameterized by spherical polar coordinates: $R_{i}, \theta_{i}, \phi_{i}, i=1, \ldots, N-1$. Then a body-fixed coordinate system is introduced, defined by aligning the body-fixed $z$ axis along the first vector, and placing the second vector in the body-fixed $x z$ plane. The remaining angles $\theta_{i}, \phi_{i}$ are either referenced with respect to the body-fixed $z$ axis, or other vectors. [6]

These coordinates are very useful, but are clearly not the most general choices. One choice of coordinates that is not treated is well known to be useful for triatomics. Sutcliffe and Tennyson[7] give the kinetic energy operator for triatomics using polyspherical coordinates in a very general form, and one finds that their expression having the body-fixed $z$ axis lying between the two vectors to be very useful. For example, taking the body-fixed $z$ axis to bisect the $\mathrm{HOH}$ angle in water gives quite good separation between rotation and vibration. One can see that this type of embedding might also be useful for systems of more than three atoms. Furthermore, for tetraatomics, it can be useful to have one of the internal angles giving the relative position of two light atoms, while another vector defines the body-fixed $z$ axis, e.g. having the $\mathrm{HCH}$ angle in formaldahyde a coordinate while the heavy $\mathrm{CO}$ group defines the body-fixed $z$ axis. In addition, it may be desirable in this example to define the orientation of $\mathrm{HCH}$ as a unit vector relative to $\mathrm{CO}$. While the first is possible using Mladenović's[6] local axis coordinates, the second is not. Ir. this work we will present a method for the derivation of kinetic energy operators for coordinates that encompass all of these choices.

The method avoids complex intermediate triganometric expresions by factorizing out the dependence on Euler angles, which is an alternative to the scheme of Lukka,[8] and allows one to build up the kinetic energy operator for complex systems from fragements where the kinetic energy operator is already known. The treatment of non-orthogonal vectors is also straightforword as is the introduction of Eckart rotations. We only consider three, four, and five atom system in the present work, but the techniques described can be easily used to obtain the kinetic energy operator for more complex systems. 


\section{INTERNAL COORDINATES AND GENERAL PLAN}

Let $\mathrm{X}$ denote the $3 \times N$ matrix of nuclear coordinates, and $\mathrm{x}$ the $3 \times N$ matrix of internal vectors, with the last vector giving the position of the nuclear center of mass. Then these vectors are related by

$$
\mathrm{X}=\mathrm{xM}
$$

with $\mathrm{M}$ an $N \times N$ matrix of mass factors. We make two resictions on $\mathrm{M}$ : first of all its inverse, denoted $\tilde{M}$, must exist, and secondly, there is no coupling of the other vectors to the nuclear center of mass vector. For a more in-depth discussion of these mass factors, see the work of Aquilanti and Cavalli,[9], Bramley and Carrington,[10] and Schwenke.[11] Then since the exact kinetic energy operator for the nuclei is

$$
T=-\frac{\hbar^{2}}{2} \sum_{\alpha} \frac{1}{m_{\alpha}} \sum_{i} \frac{\partial^{2}}{\partial X_{i \alpha}^{2}}
$$

where $m_{\alpha}$ is the mass of nuclei $\alpha$, and $i$ runs over $x, y$, and $z$, we find that

$$
T=-\frac{\hbar^{2}}{2} \sum_{\beta \beta^{\prime}}-\frac{1}{\mu_{\beta \beta^{\prime}}} \sum_{i} \frac{\partial^{2}}{\partial x_{i \beta} \partial x_{i \beta^{\prime}}},
$$

where the reduced mass factors are given by

$$
\frac{1}{\mu_{\beta \beta^{\prime}}}=\sum_{\alpha} \tilde{M}_{\alpha \beta} \tilde{M}_{\alpha \beta^{\prime}} \frac{1}{m_{\alpha}} .
$$

One can choose $M$ so that $\frac{1}{\mu_{\beta \beta^{\prime}}}$ is non-zero only when $\beta=\beta^{\prime}$, but that is not a requirement of our theory. So far we have done nothing new.

We next re-write the kinetic energy operator in the symmetric form

$$
T=\frac{\hbar^{2}}{2} \sum_{i \beta \beta^{\prime}}\left(\frac{\partial}{\partial x_{i \beta}}\right)^{\dagger} \frac{1}{\mu_{\beta \beta^{\prime}}} \frac{\partial}{\partial x_{i \beta^{\prime}}}
$$

where $\dagger$ means complex conjugate acts to the left. Since the center of mass decouples from the rest of the internal coordinates, in the sum we will take $\beta$ and $\beta^{\prime}$ to run from 1 to $N-1$.

We then seek expressions of the form

$$
\frac{\partial}{\partial x_{i \beta}}=\sum_{j} A_{j i}\left(\alpha^{L B}, \beta^{L B}, \gamma^{L B}\right) \sum_{l} g_{j k l} \hat{O}_{l}
$$

with $\mathrm{A}$ a rotation matrix parameterized by the Euler angles $\alpha^{L B}$, $\beta^{L B}$, and $\gamma^{L B}$, which take the laboratory frame to the body-frame, $\mathbf{g}$ is a matrix clepending only on vibrational 
coordinates, and the $\hat{O}_{l}$ are operators which include derivatives with respect to vibrational coordinates as well as total angular momentum components. Then, when we substitute this into Eq. 5, we obtain

$$
T=\sum_{l^{\prime}} \hat{O}_{l}^{\dagger} G_{l l^{\prime}} \hat{O}_{l^{\prime}}
$$

where

$$
G_{l l^{\prime}}=\hbar^{2} / 2 \sum_{j \beta \beta^{\prime}} g_{j \beta l}^{*} g_{j \beta^{\prime} l^{\prime}} / \mu_{\beta \beta^{\prime}}
$$

Thus once we know $g$, we can very easily form $\mathbf{G}$ and hence the kinetic energy operator.

To solve for ro-vibrational wave functions, we use the variational principle and analytic basis functions. Thus we can make explicit use of the symmetric form of the kinetic energy operator. For users of grid methods, it is required that both derivatives act to the right. However it is an easy excercise in differential calculus to convert the symmetric expressions to the more customary form. When doing this, one must bear in mind that in matrix elements of the kinetic energy operator of Eq. 7, none of the operators act on the Jacobian determinant. However, when one integrates by parts to obtain the expression with both derivatives acting to the right, the jacobian determinant is differentiated.

\section{ONE VECTOR}

Consider a single vector. Its spherical polar form in the space fixed axis system is

$$
\vec{x}=\left(\begin{array}{c}
R \sin \theta \cos \phi \\
R \sin \theta \sin \phi \\
R \cos \theta
\end{array}\right)
$$

The associated angular mometum vector is given by

$$
\vec{L}=i \hbar\left(\begin{array}{c}
\sin \phi \frac{\partial}{\partial \theta}+\cot \theta \cos \phi \frac{\partial}{\partial \phi} \\
-\cos \phi \frac{\partial}{\partial \theta}+\cot \theta \sin \phi \frac{\partial}{\partial \phi} \\
-\frac{\partial}{\partial \phi}
\end{array}\right)
$$

We will define a body-fixed $z$ axis to be along $\vec{x}$, thus to rotate vectors to this frame of reference, we will rotate by the rotation matrix $\mathbf{A}(\phi, \theta, 0)$ (see Edmonds[12] and Ref.11 for rotation conventions), i.e.

$$
\vec{x}^{b f}=\mathbf{A}(\phi, \theta, 0) \vec{x}=\left(\begin{array}{l}
0 \\
0 \\
R
\end{array}\right)
$$


and

$$
\vec{L}^{b f}=\mathbf{A}(\phi, \theta, 0) \vec{L}=i \hbar\left(\begin{array}{c}
\csc \theta \frac{\partial}{\partial \phi} \\
-\frac{\partial}{\partial \theta} \\
0
\end{array}\right)
$$

Now $\vec{L}^{b f}$ is a rather particular angular mometum vector in that it does satisfy customary commutation relations, normal or anomolous, so one must exercise care in its use. One can easily show that

$$
\frac{\partial}{\partial x_{i}}=\sum_{j} A_{j i}(\phi, \theta, 0) \sum_{l} g_{j l}^{(1)} \hat{O}_{l}
$$

with $\mathbf{g}^{(1)}$ given in Table I, and $\hat{O}_{l}$ made up of $\frac{\partial}{\partial R}, L_{x}^{b f}$, and $L_{y}^{b f}$. This expression can be substituted into Eq. 5 to yield the kinetic energy operator:

$$
T=\frac{\hbar^{2}}{2}\left(\frac{\partial}{\partial R}\right)^{\dagger} \frac{1}{\mu} \frac{\partial}{\partial R}+\left(L_{x}^{b f}\right)^{\dagger} \frac{1}{2 \mu R^{2}} L_{x}^{b f}+\left(L_{y}^{o f}\right)^{\dagger} \frac{1}{2 \mu R^{2}} L_{y}^{b f} .
$$

Substituting Eq. 12 into this yields the well known kinctic energy for a diatomic written in symmetric form.

\section{TWO VECTORS}

In this case Eq. 13 becomes

$$
\frac{\partial}{\partial x_{i \beta}}=\sum_{j} A_{j i}\left(\phi_{\beta}, \theta_{\beta}, 0\right) \sum_{l_{\beta}} g_{j l_{\beta}}^{(1)} \hat{O}_{l, 3}
$$

where $\hat{O}_{l_{1}}$ comprises $\frac{\partial}{\partial R_{1}}, L_{x_{1}}^{b f 1}, L_{y_{1}}^{b f 1}$, and $\hat{O}_{l_{2}}$ comprises $\frac{\partial}{\partial R_{2}}, L_{x_{2}}^{b f 2}$, and $L_{y_{2}}^{b f 2}$, where the superscripts on the angular momentum operators indicates they are expressed as in Eq. 12 in the axis system particular to each vector. If our internal coordinates are orthogonal, i.e. $1 / \mu_{\beta \beta^{\prime}}=0$ when $\beta \neq \beta^{\prime}$, then we can substitute this into Eq. 5 and simply obtain the kinetic energy operator for two uncoupled diatomics. This is of course only of passing interest. To take advantage of the great simplification of total angular momentum coupling or to treat non-orthogonal coordinates, we need to remove the $\beta$ dependence on the Euler angles in Eq. 15. When this is done, when we insert the expression for the derivative into Eq. 5, the rotation matrices vanish because of orthogonality. To do this we need to define a body-fixed coordinate system. This is done by a sequence of three rotations. We first rotate by $\mathbf{A}\left(\phi_{1}, \theta_{1}, 0\right)$, which takes the first vector to the body-fixed $z$ axis and the second vector to the angles $\tilde{\theta}_{2}$ and $\tilde{\phi}_{2}$. The second rotation is by $\mathbf{A}\left(0,0, \tilde{\phi}_{2}\right)$, which takes the second vector to 
the $x-z$ plane. The final rotation is $\mathbf{A}\left(0, a \tilde{\theta}_{2}, 0\right)$, where $a$ is a parameter running from zero to one.[7] When $a=0$, the body-fixed $z$ axis is along the first vector, while when $a=1$, the body-fixed $z$ axis is along the second vector, and when $a=1 / 2$, the body-fixed $z$ axis bisects the two vectors.

We begin by considering only the first two rotations. We have

$$
\begin{array}{r}
\frac{\partial}{\partial x_{i 1}}=\sum_{j} A_{j i}\left(\phi_{1}, \theta_{1}, 0\right) \sum_{l_{1}} g_{j l_{1}}^{(1)} \hat{O}_{l_{1}} \\
=\sum_{j} A_{j i}\left(\phi_{1}, \theta_{1}, \tilde{\phi}_{2}\right) \sum_{k} A_{j k}\left(0,0, \tilde{\phi}_{2}\right) \sum_{l_{1}} g_{k l_{l}}^{(1)} \hat{O}_{l_{1}} \\
=\sum_{j} A_{j i}\left(\phi_{1}, \theta_{1}, \tilde{\phi}_{2}\right) \sum_{l_{1}} g_{j l_{1}}^{(1)} \hat{\tilde{O}}_{l_{1}}
\end{array}
$$

where $\hat{\tilde{O}}_{l_{1}}$ is the same as $\hat{O}_{l_{1}}$ except that the angular momentum operators have been rotated to the body frame of the triatomic. These will be denoted $L_{x 1}^{B F}$ and $L_{y 1}^{B F}$. For the second vector we have

$$
\begin{array}{r}
\frac{\partial}{\partial x_{i 2}}=\sum_{j} A_{j i}\left(\phi_{2}, \theta_{2}, 0\right) \sum_{l_{2}} g_{j l_{2}}^{(1)} \hat{O}_{l_{2}} \\
=\sum_{j} A_{j i}\left(\phi_{1}, \theta_{1}, 0\right) \sum_{k} A_{k j}\left(\tilde{\phi}_{2}, \tilde{\theta}_{2}, 0\right) \sum_{l_{2}} g_{n l_{2}}^{(1)} \hat{O}_{l_{2}} \\
=\sum_{j} A_{j i}\left(\phi_{1}, \theta_{1}, \tilde{\phi}_{2}\right) \sum_{k} A_{k j}\left(0, \tilde{\theta}_{2}, 0\right) \sum_{l_{2}} g_{r l_{2}}^{(1)} \hat{O}_{l_{2}}
\end{array}
$$

To complete the transformation, we need to express the angular momentum operators in terms of $\frac{\partial}{\partial \tilde{\theta}_{2}}$ and the total angular momentum operators. We start by eliminating $L_{x 1}^{B F}$ and $L_{y 1}^{B F}$ by introducing the total angular momentum vector $\vec{J}$. We have to be careful here. In the space fixed frame, $\vec{J}=\vec{L}_{1}+\vec{L}_{2}$, however we have $\vec{L}_{i}$ in the frame $B F$ obtained by rotating by $\mathbf{A}\left(\phi_{1}, \theta_{1}, \tilde{\phi}_{2}\right)$. If we rotate the space frame $\vec{J}$ by $\mathbf{A}\left(\phi_{1}, \theta_{1}, \tilde{\phi}_{2}\right)$, we obtain an angular momentum vector satisfying the anomolous commutation relations, thus it is customary to reverse the signs of the components, and we will do so here as well. Thus we have

$$
L_{i 1}^{B F}=-J_{i}^{B F}-L_{i 2}^{B F}
$$

Next we need $L_{i 2}^{B F}$. Now $L_{i 2}^{b f 1}$ is given by Eq. 10 using $\tilde{\phi}_{2}$ and $\tilde{\theta}_{22}$ as the independent variables, so we rotate $\vec{L}_{2}^{b f 1}$ by $\mathbf{A}\left(0,0, \tilde{\phi}_{2}\right)$, which yields $L_{x 2}^{B F}=i \hbar \cot \tilde{\theta}_{2} \frac{\partial}{\partial \tilde{p}_{2}}$ and $L_{y_{2}}^{B F}=-i \hbar \frac{\partial}{\partial \tilde{\theta}_{2}}$. Finally we note that

$$
J_{z}=i \hbar \frac{\partial}{\partial \tilde{\phi}_{2}}
$$


and we use this to eliminate the derivative with respect to $\tilde{\phi}_{2}$. In $\hat{O}_{l_{2}}$, we need $L_{i 2}^{b f 2}$. This is given by Eq. 12 using $\tilde{\phi}_{2}$ and $\tilde{\theta}_{2}$ as the independent variables.

In summary we now have

$$
\frac{\partial}{\partial x_{i \beta}}=\sum_{j} A_{j 2}\left(\phi_{1}, \theta_{1}, \tilde{\phi}_{2}\right) \sum_{l} \tilde{g}_{j \beta l}^{(2)} \hat{O}_{l}
$$

for $\beta=1$ and 2 , where $\tilde{\mathbf{g}}^{(2)}$ is given by Table II with $a=0$, and $\hat{O}_{l}$ comprizes $\frac{\partial}{\partial R_{1}}, \frac{\partial}{\partial R_{2}}$, $\frac{\partial}{\partial \chi}, J_{x}^{B F}, J_{y}^{B F}$, and $J_{z}^{B F}$, where $\chi=\tilde{\theta}_{2}$ is the angle between the two vectors. We can then substitute this into Eq. 8, and obtain the well known expression for the triatomic kinetic energy.[13]

For the most part, the introduction of the third rotation is straightforward. We insert a resolution of the identity so that Eq. 24 becomes

$$
\begin{array}{r}
\frac{\partial}{\partial x_{i \beta}}=\sum_{j} A_{j i}\left(\phi_{1}, \theta_{1}, \tilde{\phi}_{2}\right) \sum_{k m} A_{m j}\left(0, a \tilde{\theta}_{2}, 0\right) A_{m k}\left(0, a \tilde{\theta}_{2}, 0\right) \sum_{l} \tilde{g}_{k \beta l}^{(2)} \hat{O}_{l} \\
=\sum_{j} A_{j i}\left(\alpha^{L B}, \beta^{L B}, \gamma^{L I 3}\right) \sum_{k} A_{j k}\left(0, a \tilde{\theta}_{2}, 0\right) \sum_{l} \tilde{g}_{k \beta l}^{(2)} \hat{O}_{l}
\end{array}
$$

with $\alpha^{L B}, \beta^{L B}$, and $\gamma^{L B}$ the new Euler angles relating the space fixed frame to the new body-fixed frame denoted $\mathcal{B F}$. It is straightforward to multiply the matrices $A$ and $\tilde{g}^{(2)}$ to get a new effective $g^{(2)}$, but more care needs to be taken to reate $\vec{J}$ in body frame $B F$ to $\vec{J}$ in body frame $\mathcal{B F}$. Formally we are changing variables from $\phi_{1}, \theta_{1}, \tilde{\phi}_{2}$, and $\tilde{\theta}_{2}$ to $\alpha^{L B}, \beta^{L B}$, $\gamma^{L B}$, and $\chi$, where

$$
\mathbf{A}\left(\alpha^{L B}, \beta^{L B}, \gamma^{L B}\right)=\mathbf{A}\left(\phi_{1}, \theta_{1}, \tilde{\phi}_{2}\right) \mathbf{A}\left(0 . a \tilde{\theta}_{2}, 0\right)
$$

and

$$
\chi=\tilde{\theta}_{2}
$$

Using the chain rule, we have

$$
\frac{\partial}{\partial \omega}=\frac{\partial \alpha^{L B}}{\partial \omega} \frac{\partial}{\partial \alpha^{L B}}+\frac{\partial \beta^{L B}}{\partial \omega} \frac{\partial}{\partial \beta^{L B}}+\frac{\partial \gamma^{L B}}{\partial \omega} \frac{\partial}{\partial \gamma^{L B}}+\frac{\partial \chi^{L B}}{\partial \omega} \frac{\partial}{\partial \chi^{L B}}
$$

$\omega=\phi_{1}, \theta_{1}, \tilde{\phi}_{2}$, and $\tilde{\theta}_{2}$. Now the only nonzero $\frac{\partial \chi^{L B}}{\partial \omega}$ occurs for $\omega=\tilde{\theta}_{2}$, thus the equations for $\omega \neq \tilde{\theta}_{2}$ are just the equations describing a rotation by the constant $a \tilde{\theta}_{2}$, thus

$$
\vec{J}^{\mathcal{B F}}=\mathbf{A}\left(0, a \tilde{\theta}_{2}, 0\right) \vec{J}^{B F}
$$

can be used to eliminate the $B F$ components of the total angular momentum in terms of the $\mathcal{B F}$ components. This amounts to multiplying the final three columns of the result so 
far from the right by $\mathbf{A}^{T}\left(0, a \tilde{\theta}_{2}, 0\right)$. The final equation involving $\tilde{\theta}_{2}$ is most easily analyzed considering the instantenous case where $\phi_{1}=\theta_{1}=\tilde{\phi}_{2}=0$. Then we have

$$
\frac{\partial}{\partial \tilde{\theta}_{2}}=-a i J_{y}^{\mathcal{B F}}+\frac{\partial}{\partial \chi}
$$

i.e. the fifth column of the result so far needs to be aumented by --ai times the third column. Combining all of this together, we have

$$
\frac{\partial}{\partial x_{i \beta}}=\sum_{j} A_{j i}\left(\alpha^{L B}, \beta^{L B}, \gamma^{L B}\right) \sum_{l} g_{j \beta l}^{(2)} \hat{O}_{l}
$$

with $\mathbf{g}^{(2)}$ given by Table II and $\hat{O}_{l}$ comprizing $\frac{\partial}{\partial R_{1}}, \frac{\partial}{\partial R_{2}}, \frac{\partial}{\partial \chi}, J_{x}^{\mathcal{B F}}, J_{y}^{\mathcal{B F}}$, and $J_{z}^{\mathcal{B F}}$. Substituting this expression into Eq. 5 we obtain the expression of Sutcliffe and Tennyson[7] for their $z$-axis embedding coordinates.

It should be noted that $\mathbf{g}^{(2)}$ in Table II is overly complex for triatomics. This is because we could have factored out the rotation matrix left multiplying $\tilde{\mathbf{g}}^{(2)}$, and this rotation matrix would collapse down to the unit matrix in Eq. 8. However this rotation matrix is required for the next sections, so we retain it here as well.

To obtain the $x$-axis embedding of Sutcliffe and Tennysor, we simply insert a rotation that exchanges the $x$ and $z$ axes. This changes $\mathbf{g}^{(2)}$ in only tc a minor extent. We can also exchange the $z$ and $y$ axis, if desired.[14]

Now consider the possibility that $a$ depends on $R_{1}, R_{2}$, and $\chi$. The only changes are Eq. 31 is replaced by

$$
\frac{\partial}{\partial \tilde{\theta}_{2}}=-\left(a+\chi \frac{\partial a}{\partial \chi}\right) i J_{y}^{\mathcal{B F}}+\frac{\partial}{\partial \chi}
$$

and

$$
\frac{\partial}{\partial R_{i}} \rightarrow \frac{\partial}{\partial R_{i}}-i \chi \frac{\partial a}{\partial R_{i}} J_{y}^{\mathcal{B F}},
$$

where the arrow means "replaced by", and these changes modify $g^{(2)}$ in a straightforward manner. That is, it is not difficult to choose $a$ so that the Eckart conditions[15] separating vibration and rotation are satisfied. The only complication is the kinetic energy operator matrix elements are no longer obtainable analytically, but it should be possible to obtain accurate results numerically with very little additional cost compared to calculations keeping $a$ fixed. There is, of course, still the problem of singularities arising when the Eckart conditions lead to ill posed equations at linear configurations. 


\section{THREE VECTORS}

When we have three vectors, we use the results from the single vector case and the two vector case. We still use the notation $\vec{J}^{\mathcal{B F}}$ for the total angular momentum in the final body-fixed frame, so we will change our notation for the two vector result. For the two vector sub-part, we will call the angular momentum $\vec{j}_{12}$, the body-fixed frame defined for it bf 12 , and the Euler angles $\alpha_{12}, \beta_{12}$, and $\gamma_{12}$. Then we have

$$
\frac{\partial}{\partial x_{i \beta}}=\sum_{j} A_{j i}\left(\alpha_{12}, \beta_{12}, \gamma_{12}\right) \sum_{l_{12}} g_{j \beta l_{12}}^{(2)} \hat{O}_{l_{12}}
$$

for $\beta=1$ and 2 , with $\hat{O}_{l_{12}}$ comprizing $\frac{\partial}{\partial R_{1}}, \frac{\partial}{\partial R_{2}}, \frac{\partial}{\partial \chi}, j_{x}^{b f 12}, j_{y}^{b f 12}$, and $j_{z}^{b f 12}$, and

$$
\frac{\partial}{\partial x_{i 3}}=\sum_{j} A_{j i}\left(\phi_{3}, \theta_{3}, 0\right) \sum_{l_{3}} g_{j l_{3}}^{(1)} \hat{O}_{l_{3}}
$$

with $\hat{O}_{l_{3}}$ comprizing $\frac{\partial}{\partial R_{3}}, L_{x 3}^{b f 3}$, and $L_{y 3}^{b f 3}$.

Next we have two choices. We can make the over-all Euler angles $\phi_{3}, \theta_{3}$, and $\tilde{\alpha}_{12}$, where $\tilde{\alpha}_{12}$ is defined by

$$
\mathbf{A}\left(\tilde{\alpha}_{12}, \tilde{\beta}_{12}, \tilde{\gamma}_{12}\right)=\mathbf{A}\left(\alpha_{12}, \beta_{12}, \gamma_{12}\right) \mathbf{A}^{T}\left(\phi_{3}, \theta_{3}, 0\right)
$$

or we could make the over-all Euler angles $\alpha_{12}, \beta_{12}$, and $\gamma_{12}$. It is equally easy to derive the matrix $g^{(3)}$ for each case, but for illustration in this section, we consider the former case. This is also the situation considered in Ref.11, where it was called the $l j k$ coupling scheme. The later case would be treated as in the next section.

Thus we follow the procedure of the previous section to obtain

$$
\frac{\partial}{\partial x_{i \beta}}=\sum_{j} A_{j i}\left(\phi_{3}, \theta_{3}, \tilde{\alpha}_{12}\right) \sum_{k} A_{k j}\left(0, \tilde{\beta}_{12}, \tilde{\gamma}_{12}\right) \sum_{i_{12}} g_{k \beta l_{12}}^{(2)} \hat{O}_{l_{12}}
$$

for $\beta=1$ and 2 , and for $\beta=3$,

$$
\frac{\partial}{\partial x_{i 3}}=\sum_{j} A_{j i}\left(\phi_{3}, \theta_{3}, \tilde{\alpha}_{12}\right) \sum_{l_{3}} g_{j l_{3}}^{(1)} \tilde{\tilde{O}}_{i 3}
$$

where $\hat{\tilde{O}}_{l_{3}}$ comprizes $\frac{\partial}{\partial R_{3}}, L_{x 3}^{B F}$, and $L_{y 3}^{B F}$, where $B F$ is the body-fixed frame defined by the Euler angles $\phi_{3}, \theta_{3}$, and $\tilde{\alpha}_{12}$. We then introduce the total angular momentum $\vec{J}^{B F}$ to eliminate $\vec{L}_{3}^{B F}$ :

$$
\vec{L}_{3}^{B F}=-\vec{J}^{B F}-\vec{j}_{12}^{B F}
$$


The functional form of $\vec{j}_{12}^{B F}$ is given by rotating $\vec{j}_{12} f 3$ by $\tilde{\alpha}_{12}$ :

$$
\vec{j}_{12}^{B F}=\mathbf{A}\left(0,0, \tilde{\alpha}_{12}\right) \vec{j}_{12}^{b f 3}=i \hbar\left(\begin{array}{c}
\cot \tilde{\beta}_{12} \frac{\partial}{\partial \tilde{\alpha}_{12}}-\csc \tilde{\beta}_{12} \frac{\partial}{\partial \tilde{\gamma}_{12}} \\
-\frac{\partial}{\partial \tilde{\beta}_{12}} \\
-\frac{\partial}{\partial \tilde{\alpha}_{12}}
\end{array}\right),
$$

where

$$
\vec{j}_{12}^{b f 3}=-i \hbar\left(\begin{array}{c}
-\cos \tilde{\alpha}_{12} \cot \tilde{\beta}_{12} \frac{\partial}{\partial \tilde{\alpha}_{12}}-\sin \tilde{\alpha}_{12} \frac{\partial}{\partial \tilde{\beta}_{12}}+\cos \tilde{\alpha}_{12} \csc \tilde{\beta}_{12} \frac{\partial}{\partial \tilde{\gamma}_{12}} \\
-\sin \tilde{\alpha}_{12} \cot \tilde{\beta}_{12} \frac{\partial}{\partial \tilde{\alpha}_{12}}+\cos \tilde{\alpha}_{12} \frac{\partial}{\partial \tilde{\beta}_{12}}+\sin \tilde{\alpha}_{12} \csc \tilde{\beta}_{12} \frac{\partial}{\partial \tilde{\gamma}_{12}} \\
\frac{\partial}{\partial \tilde{\alpha}_{12}}
\end{array}\right) .
$$

We also use

$$
\vec{j}_{12}^{b f 12}=i \hbar\left(\begin{array}{c}
-\cos \tilde{\gamma}_{12} \csc \tilde{\beta}_{12} \frac{\partial}{\partial \tilde{\alpha}_{12}}+\sin \tilde{\gamma}_{12} \frac{\partial}{\partial \bar{\beta}_{12}}+\cos \tilde{\gamma}_{12} \cot \tilde{\beta}_{12} \frac{\partial}{\partial \bar{\gamma}_{12}} \\
\sin \tilde{\gamma}_{12} \csc \tilde{\beta}_{12} \frac{\partial}{\partial \tilde{\alpha}_{12}}+\cos \tilde{\gamma}_{12} \frac{\partial}{\partial \tilde{\beta}_{12}}-\sin \tilde{\gamma}_{12} \cot \tilde{\beta}_{12} \frac{\partial}{\partial \tilde{\gamma}_{12}} \\
\frac{\partial}{\partial \tilde{\gamma}_{12}}
\end{array}\right)
$$

to transform to differential expressions. Finally, we eliminate $\frac{\partial}{\partial \tilde{\alpha}_{12}}$ by using

$$
J_{z}^{B F}=i \hbar \frac{\partial}{\partial \tilde{\alpha}_{12}}
$$

In summary, we obtain

$$
\frac{\partial}{\partial x_{i \beta}}=\sum_{j} A_{j i}\left(\phi_{3}, \theta_{3}, \tilde{\alpha}_{12}\right) \sum_{l} \tilde{g}_{j \beta l}^{(3)} \hat{C}_{l},
$$

where $\tilde{g}_{j 3 l}^{(3)}$ is given by

$$
\tilde{g}_{j \beta l}^{(3)}=\left\{\begin{array}{ll}
\sum_{k} A_{j k}\left(0, \tilde{\beta}_{12}, \tilde{\gamma}_{12}\right) \bar{g}_{k \beta l}^{(3)} & \beta=1,2 \\
\bar{g}_{j \beta l}^{(3)} & \beta=3
\end{array},\right.
$$

with $\bar{g}_{j \beta l}^{(3)}$ given in Table III, and $\hat{O}_{l}$ comprizing $\frac{\partial}{\partial R_{1}}, \frac{\partial}{\partial R_{2}}, \frac{\partial}{\partial R_{3}}, \frac{\partial}{\partial \chi}, \frac{\partial}{\partial \dot{\beta}_{12}}, \frac{\partial}{\partial \tilde{\gamma}_{12}}, J_{x}^{B F}, J_{y}^{B F}$, and $J_{z}^{B F}$. It should be noted that when orthogonal coordinates are used, the rotation matrices $\mathbf{A}\left(0, \tilde{\beta}_{12}, \tilde{\gamma}_{12}\right)$ will collapse to give the unit matrix when inserted into Eq. 8. Only for non-orthogonal coordinates do these give contributions to the functional form of the kinetic energy operator.

Next let us re-address the issue of using $\phi_{3}, \theta_{3}$, and $\tilde{\alpha}_{12}$ rather than $\alpha_{12}, \beta_{12}$, and $\gamma_{12}$ as the overall Euler angles. Since one can show that (see Eq. 37)

$$
\mathbf{A}\left(0, \tilde{\beta}_{12}, \tilde{\gamma}_{12}\right) \mathbf{A}\left(\phi_{3}, \theta_{3}, \tilde{\alpha}_{12}\right)=\mathbf{A}\left(\alpha_{12}, \beta_{12}, \gamma_{12}\right)
$$


if we introduce the rotation $\mathbf{A}\left(0, b \tilde{\beta}_{12}, b \tilde{\gamma}_{12}\right)$, then when $b=0$, we recover the $\phi_{3}, \theta_{3}, \tilde{\alpha}_{12}$ case, while when $b=1$, we recover the $\alpha_{12}, \beta_{12}, \gamma_{12}$ case. This is exactly analogous to the rotation $\mathbf{A}\left(0, a \tilde{\theta}_{2}, 0\right)$ introduced in the previous section. Ther. we have

$$
\frac{\partial}{\partial x_{i \beta}}=\sum_{j} A_{j i}\left(\alpha^{L B}, \beta^{L B}, \gamma^{L B}\right) \sum_{k} A_{j k}\left(0, b \tilde{\beta}_{12}, t \tilde{\gamma}_{12}\right) \sum_{l} \tilde{g}_{k \beta l}^{(3)} \hat{O}_{l}
$$

where $\alpha^{L B}, \beta^{L B}, \gamma^{L B}$ define the body-frame $\mathcal{B F}$. As before, we have

$$
\vec{J}^{\mathcal{B F}}=\mathbf{A}\left(0, b \tilde{\beta}_{12}, b \tilde{y}_{12}\right) \vec{J}^{B F}
$$

but now

$$
\frac{\partial}{\partial \tilde{\beta}_{12}} \rightarrow-i b \sin b \tilde{\beta}_{12} J_{x}^{\mathcal{B F}}-i b \cos b \tilde{\beta}_{12} J_{y}^{\mathcal{B} \mathcal{F}}+\frac{\partial}{\partial \tilde{\beta}_{12}}
$$

and

$$
\frac{\partial}{\partial \tilde{\gamma}_{12}} \rightarrow-i b J_{z}^{\mathcal{B F}}+\frac{\partial}{\partial \tilde{\gamma}_{12}}
$$

We determined these last two relations by numerical experimentation. Thus we multiply $\tilde{\mathbf{g}}^{(3)}$ from the left by $\mathbf{A}\left(0, b \tilde{\beta}_{12}, b \tilde{\gamma}_{12}\right)$, multiply the final three columns of the result from the right by $\mathrm{A}^{T}\left(0, b \tilde{\beta}_{12}, b \tilde{\gamma}_{12}\right)$, then subtract $i b \sin b \tilde{\gamma}_{12}$ times the fifth column from the seventh column, subtract $i b \cos b \tilde{\gamma}$ times the fifth column from the eighth column, and subtract $i b$ times the sixth column from the ninth column to obtain the matrix $\mathbf{g}^{(3)}$ in the expression

$$
\frac{\partial}{\partial x_{i \beta}}=\sum_{j} A_{j i}\left(\alpha^{L B}, \beta^{L B}, \gamma^{L B}\right) \sum_{l} g_{j \beta l}^{(i)} \hat{O}_{l}
$$

where $\hat{O}_{l}$ comprizes $\frac{\partial}{\partial R_{1}}, \frac{\partial}{\partial R_{2}}, \frac{\partial}{\partial R_{3}}, \frac{\partial}{\partial \chi}, \frac{\partial}{\partial \tilde{\beta}_{12}}, \frac{\partial}{\partial \tilde{\gamma}_{12}}, J_{x}^{\mathcal{B F}}, J_{y}^{\mathcal{B F}}$, and $J_{z}^{\mathcal{B F}}$.

It should be noted that the rotation by $b \tilde{\beta}_{12}, b \tilde{\gamma}_{12}$ has no effect on the kinetic energy for $J=0$.

Kinetic energy operators using the embedding defined by $b$ have never been used before, and it would be very interesting to explore the benefits that arise from allowing $b$ to be different from 0 or 1 . One example that immediately comes to mind is this allows the $z$ axis to be aligned with the symmetry axis for $\mathrm{XH}_{3}$ molecules at the equilibrium geometry.

Although we do not do so here, it should be straightforward to introduce Eckart rotations at this point. However, in contrast to the two vector case, it will not be possible to satisfy the Eckart relations by varying a single parameter. 


\section{FOUR VECTORS}

When we have four vectors, one can use the results of a single vector and the results of three vectors, or one can use the results for two vectors twice. The former case is exactly analogous to the three vector case treated above. Following the pattern given before, one can easily derive the appropriate $\mathbf{g}^{(4)}$ for either case. Since $\mathrm{CH}_{4}$ is a molecule of interest to us, we will only consider the case when we use two vectors twice.

As in the three vector case, we need to change the two vector notation. For the first two vector sub-part, we will call the angular momentum $\vec{j}_{12}$, the body-fixed frame defined for it $b f 12$, and the Euler angles $\alpha_{12}, \beta_{12}$, and $\gamma_{12}$. The quantities for the second two vector sub-part are defined analogously, except with the subscript 34 . We will take $\alpha_{12}, \beta_{12}$, and $\gamma_{12}$ to define the body-fixed frame $B F(=b f 12)$, so that we have for $\beta=1$ and 2 ,

$$
\frac{\partial}{\partial x_{i \beta}}=\sum_{j} A_{j i}\left(\alpha_{12}, \beta_{12}, \gamma_{12}\right) \sum_{l_{12}} g_{j \beta l_{12}}^{(2)} \hat{O}_{l_{12}}
$$

with $\hat{O}_{l_{12}}$ comprizing $\frac{\partial}{\partial R_{1}}, \frac{\partial}{\partial R_{2}}, \frac{\partial}{\partial \chi_{12}}, j_{x 12}^{B F}, j_{y 12}^{B F}$, and $j_{z 12}^{B F}$, and for $\beta=3$ and 4 ,

$$
\frac{\partial}{\partial x_{i \beta}}=\sum_{j} A_{j i}\left(\alpha_{12}, \beta_{12}, \gamma_{12}\right) \sum_{k} A_{k j}\left(\tilde{\alpha}_{34}, \tilde{\beta}_{34}, \tilde{\gamma}_{34}\right) \sum_{l_{34}} g_{k \beta l_{34}}^{(2)} \hat{O}_{l_{34}},
$$

with $\hat{O}_{l_{34}}$ comprizing $\frac{\partial}{\partial R_{3}}, \frac{\partial}{\partial R_{4}}, \frac{\partial}{\partial \chi_{34}}, j_{x 34}^{b f 34}, j_{y 34}^{b f 34}$, and $j_{z 34}^{b f 34}$, with

$$
\mathbf{A}\left(\tilde{\alpha}_{34}, \tilde{\beta}_{34}, \tilde{\gamma}_{34}\right)=\mathbf{A}\left(\alpha_{34}, \beta_{34}, \gamma_{34}\right) \mathbf{A}^{T}\left(\alpha_{12}, \beta_{12}, \gamma_{12}\right)
$$

We proceed as before by introducing the total angular momentum, which because $\vec{j}_{12}^{B F}$ and $\vec{J}^{B F}$ are sign reversed while $\vec{j}_{34}^{B F}$ is not, yeilds

$$
\vec{j}_{12}^{B F}=\vec{J}+\vec{j}_{34}^{B F}
$$

However $\vec{j}_{34}^{B F}$ is given by Eq. 42 , so in the case of orthogonal coordinates where the rotation $\mathbf{A}\left(\tilde{\alpha}_{34}, \tilde{\beta}_{34}, \tilde{\gamma}_{34}\right)$ vanishes in the kinetic energy operator due to orthogonality, we obtain a quite simple expression in terms of the 15 operators $\frac{\partial}{\partial R_{1}}, \frac{\partial}{\partial R_{2}}, \frac{\partial}{\partial R_{3}}, \frac{\partial}{\partial R_{4}}, \frac{\partial}{\partial \chi_{12}}, \frac{\partial}{\partial \chi_{34}}, j_{x 34}^{B F}$. $j_{y 34}^{B F}, j_{z 34}^{B F}, j_{x 34}^{b f 34}, j_{y 34}^{b f 34}, j_{z 34}^{b f 34}, J_{x}^{B F}, J_{y}^{B F}$, and $J_{z}^{B F}$. We can use both Eq. 42 and Eq. 43 to convert $j_{x 34}^{B F}$ etc to derivatives with respect to $\tilde{\alpha}_{34}, \tilde{\beta}_{34}$, and $\tilde{\gamma}_{34}$, so there are only 12 unique operators.

In Table IV we give the resulting matrix in symbolic form. There it is understood that the matrices $\mathbf{g}^{(2.1)}$ are the first three columns of $\mathbf{g}^{(2)}, \mathbf{g}^{(2.2)}$ are the last three columns of $\mathbf{g}^{(2)}$, and first three rows are for $\beta=1$, the next for $\beta=2$, etc. 
However the kinetic energy operator derived in this way does not couple vibration and rotation in a symmetric manner. This is analogous to the triatomic case when one treats $\mathrm{H}_{2} \mathrm{O}$ with $a=0$. So we need to introduce the additional rotaion $\mathbf{A}\left(c \tilde{\alpha}_{34}, c \tilde{\beta}_{34}, c \tilde{\gamma}_{34}\right)$, which reverts to the above case when $c=0$, makes the overall Euler angles $\alpha_{34}, \beta_{34}, \gamma_{34}$ when $c=1$, and treats the two pairs of vectors more equivalently when $c=1 / 2$. This rotation is included exactly as before, with the analog of relations 50 and 51 being

$$
\begin{gathered}
\frac{\partial}{\partial \tilde{\alpha}_{34}} \rightarrow-i c\left(-\sin c \tilde{\beta}_{34} \cos c \tilde{\gamma}_{34} J_{x}^{\mathcal{B F}}+\sin c \tilde{\beta}_{34} \sin c \tilde{\gamma}_{34} J_{y}^{\mathcal{B F}}+\cos c \tilde{\beta}_{34} J_{z}^{\mathcal{B F}}\right)+\frac{\partial}{\partial \tilde{\alpha}_{34}} \\
\frac{\partial}{\partial \tilde{\beta}_{34}} \rightarrow-i c\left(\sin c \tilde{\gamma}_{34} J_{x}^{\mathcal{B F}}+\cos c \tilde{\gamma}_{34} J_{y}^{\mathcal{B F}}\right)+\frac{\partial}{\partial \tilde{\beta}_{34}}
\end{gathered}
$$

and

$$
\frac{\partial}{\partial \tilde{\gamma}_{34}} \rightarrow-i c J_{z}^{\mathcal{B F}}+\frac{\partial}{\partial \tilde{\gamma}_{34}}
$$

When one considers cases where $c$ is nonzero, the resulting expressions can become quite complex. Since we are interested in including non-adiabatic corrections which in general requires numerical techniques,[17] we will take a numerical approach to evaluate matrix elements of the kinetic energy operator. In order to do this, one has to take care that the singularities are treated properly. We do this by writing

$$
\mathbf{g}^{(4)}=\sum_{s} \mathbf{g}_{s}^{(4)} f_{s}
$$

with $f_{s}$ equal to $1 / R_{1}, 1 / R_{2}, 1 / R_{3}, 1 / R_{4}, 1 / \sin \chi_{12}, 1 / \sin \chi_{34}$, and $1 / \sin \tilde{\beta}_{34}$. The $\mathbf{g}_{s}^{(4)}$ will always be finite. The we have

$$
G_{l l^{\prime}}=\hbar^{2} / 2 \sum_{s s^{\prime}} f_{s} f_{s^{\prime}} \sum_{j \beta \beta^{\prime}} g_{j \beta l s}^{*} g_{j \beta^{\prime} l^{\prime} s^{\prime}} / \mu_{\beta \beta^{\prime}}
$$

and we use appropriate basis functions and quadratures which handle the singular factors.

\section{DISCUSSION AND CONCLUSIONS}

We have discussed the procedure for producing kinetic energy operators for polyatomics using polyspherical coordinates and very general embeddings of body-fixed $z$ axes. We do this by introducing the expression

$$
\frac{\partial}{\partial x_{i \beta}}=\sum_{j} A_{j i}\left(\alpha^{L B}, \beta^{L B}, \gamma^{L B}\right) \sum_{l} g_{j, l} \hat{O}_{l}
$$


The matrices $g$ can be very easily built up from the results from fewer numbers of vectors, and the kinetic energy operator coefficients are simply given by dot products of columns of $\mathbf{g}$. Non-orthogonal coordinate systems pose no formal difficulty -..- however in practice, much more complicated expressions arise. We also show how to generalize the a parameterized axis embedding of Sutcliffe and Tennyson[7] to more than three atoms.

One motivation for this work was the derivation of the expressions for the four vector case using non-orthogonal coordinates. One may ask why one would want to use nonorthogonal coordinates since the kinetic energy operator is so much simpler for orthogonal coordinates? There are several reasons for this. One reason, put forward by Handy,[16] is that the potential matrix elements are much simpler when one uses the physical bondlength-bond-angle coordinates. While less of an issue for triatomics, this becomes very important as the number of atoms increases, especially if one wants to use a single potential energy surface for more than one isotopomer. The second reason is related to the treatment of Born-Oppenheimer breakdown. We have showed the importance of including both first and second order corrections for $\mathrm{H}_{2} \mathrm{O},[17]$ and since the second order correction gives rise to cross terms in the kinetic energy operator, even when orthogonal coordinates are used, the argument of simplicity no longer is valid. In addition, it is very useful for debugging programs to be able to compare the results obtained using different coordinates. Finally, it can be useful to turn to non-orthogonal coordinates to improve the convergence of the basis function expansion.[18]

In addition to its utility for deriving expressions for the kiretic energy operator, the matrices $\mathrm{g}$ are also required in generating non-adiabatic correction function originally computed for cartesian nuclear position vectors.[19]

In this work, we have not discussed what basis functions to use with these kinetic energy operators. Basically there are only two restrictions: first of all the functions must give finite matrix elements, and secondly they must adequately cover the Hilbert space for the problem of interest. For triatomics with $a \neq 0$, we used Jacobi polynomial basis functions to avoid the singularities in the matrix elements.[20] For the three and four vector case, it is not yet clear what changes one needs to make to ensure finite matrix elements when $b$ or $c$ are 
non-zero.

[1] M. Mladenović, J. Chem. Phys. 112, 1070 (2000).

[2] M. Mladenović, J. Chem. Phys. 112, 1082 (2000).

[3] F. Gatti, C. Iung, M. Menou, Y. Justum, A. Nauts, and X. Chapuisat, J. Chem. Phys. 108, 8804 (1998).

[4] F. Gatti, C. Iung, M. Menou, and X. Chapuisat, J. Chem. Phys. 108, 8821 (1998)

[5] F. Gatti, C. Muñoz, and C. Iung, J. Chem. Phys. 114, 8275 (2001).

[6] M. Mladenović, J. Chem. Phys. 113, 10524 (2000).

[7] B. T. Sutcliffe and J. Tennyson, Int. J. Quant. Chem. 39, 183 (1991).

[8] T. J. Lukka, J. Chem. Phys. 102, 3945 (1995).

[9] V. Aquilanti and S. Cavalli, J. Chem. Phys. 85, 1355 (1986).

[10] M. J. Bramley and T. Carrington, J. Chem. Phys. 99, 8519 (1993).

[11] D. W. Schwenke, J. Phys. Chem. 100, 2867 (1996); 100, 18884 (1996).

[12] A. R. Edmonds, "Angular Momentum in Quantum Mechanics", third printing with corrections, Princeton University Press, Princeton, 1974.

[13] R. T Pack, J. Chem. Phys. 60, 633 (1974).

[14] O. Polyanski, poster at CCP6 workshop, Manchester, UK, Dec. 2001.

[15] "Molecuar Vibrations", E. B. Wilson, J. C. Decius, and P. C. Cross, Dover Publications, Inc, New York, New York, 1955.

[16] S. Carter and N. C. Handy, Mol. Phys. 47, 1445 (1982).

[17] D. W. Schwenke, J. Phys. Chem. A, 105, 2352 (2001).

[18] D. W. Schwenke, Chem. Phys. Lett. 189, 91 (1992).

[19] D. W. Schwenke, to be published.

[20] D. W. Schwenke, Comp. Phys. Commun. 70, 1 (1992). 
TABLE I: Coordinate transformation matrix $\mathbf{g}^{(1)}$.

$$
\begin{array}{cccc}
\hline & \frac{\partial}{\partial h} & L_{x}^{b f} & L_{y}^{b f} \\
\hline \mathrm{x} & 0 & 0 & \frac{-1}{i R} \\
\mathrm{y} & 0 & \frac{1}{i R} & 0 \\
\mathrm{z} & 1 & 0 & 0
\end{array}
$$


TABLE II: Coordinate transformation matrix $\mathbf{g}^{(2)} \cdot \bar{a}=1-a$.

\begin{tabular}{cccccc}
\hline$\frac{\partial}{\partial R_{1}}$ & $\frac{\partial}{\partial \bar{R}_{2}}$ & $\frac{\partial}{\partial \chi}$ & $J_{x}$ & $J_{y}$ & $J_{z}$ \\
\hline$-\sin a \chi$ & 0 & $-\cos a \chi / r_{1}$ & 0 & $\bar{a} \cos a \chi / i r_{1}$ & 0 \\
0 & 0 & 0 & $-\sin \bar{a} \chi /\left(i r_{1} \sin \chi\right)$ & 0 & $-\cos \bar{a} \chi /\left(i r_{1} \sin \chi\right)$ \\
$\cos a \chi$ & 0 & $-\sin a \chi / r_{1}$ & 0 & $\bar{a} \sin a \chi / i r_{1}$ & 0 \\
0 & $\sin \bar{a} \chi$ & $\cos \bar{a} \chi / r_{2}$ & 0 & $a \cos \bar{a} \chi / i r_{2}$ & 0 \\
0 & 0 & 0 & $-\sin a \chi /\left(i r_{2} \sin \chi\right)$ & 0 & $\cos a \chi /\left(i r_{2} \sin \chi\right)$ \\
0 & $\cos \bar{a} \chi$ & $-\sin \bar{a} \chi / r_{2}$ & 0 & $-a \sin \bar{a} \chi / i r_{2}$ & 0
\end{tabular}


TABLE III: Coordinate transformation matrix $\overline{\mathbf{g}}^{(3)} \cdot \bar{a}=1-a$.

\begin{tabular}{|c|c|c|c|c|c|c|c|c|}
\hline$\frac{\partial}{\partial R_{1}}$ & $\frac{\partial}{\partial R_{2}}$ & $\frac{\partial}{\partial R_{3}}$ & $\frac{\partial}{\partial \chi}$ & $\frac{\partial}{\partial \overline{3}}$ & $\frac{\partial}{\partial \ddot{\gamma}}$ & $J_{x}$ & $J_{y}$ & $J_{z}$ \\
\hline \multirow[t]{2}{*}{$-\sin a \chi$} & 0 & 0 & $-\cos a \chi / r_{1}$ & $\bar{a} \cos a \chi$ & $-\bar{a} \cos a \chi \sin \tilde{\gamma} \cot \hat{\gamma} / r_{1}$ & 0 & 0 & $\bar{a} \cos a \chi \sin \tilde{\gamma}$ \\
\hline & & & & $\times \cos \tilde{\gamma} / r_{1}$ & & & & $\times \csc \tilde{\beta} / i r_{1}$ \\
\hline \multirow[t]{2}{*}{0} & 0 & 0 & 0 & $-\sin \bar{a} \chi \sin \tilde{\gamma}$ & $-[\sin \bar{a} \chi \cos \tilde{\gamma} \cot \tilde{\beta}$ & 0 & 0 & $\sin \bar{a} \chi \cos \tilde{\gamma} \csc \tilde{\beta}$ \\
\hline & & & & $x \csc \chi / r_{1}$ & $+\cos \bar{a} \chi] \csc \chi / r_{1}$ & & & $\times \csc \chi / i r_{1}$ \\
\hline \multirow[t]{2}{*}{$\cos a \chi$} & 0 & 0 & $-\sin a \chi / r_{1}$ & $\bar{a} \sin a_{\chi}$ & $-\bar{a} \sin a \chi \sin \tilde{\gamma} \cot \tilde{\beta} / r_{1}$ & 0 & 0 & $\bar{a} \sin a \chi \sin \tilde{\gamma}$ \\
\hline & & & & $\times \cos \tilde{\gamma} / r_{1}$ & & & & $\times \csc \tilde{\beta} / i r_{1}$ \\
\hline \multirow[t]{2}{*}{0} & $\sin \bar{a} \chi$ & 0 & $\cos \bar{a} \chi / r_{2}$ & $a \cos \bar{a} \chi$ & $-a \cos \bar{a} \chi \sin \tilde{\gamma} \cot \tilde{F} / r_{2}$ & 0 & 0 & $a \cos \bar{a} \chi \sin \tilde{\gamma}$ \\
\hline & & & & $\times \cos \tilde{\gamma} / r_{2}$ & & & & $\times \csc \tilde{\beta} / i r_{2}$ \\
\hline \multirow[t]{2}{*}{0} & 0 & 0 & 0 & $-\sin a \chi \sin \tilde{\gamma}$ & $-\left[\sin a_{\chi} \chi \cos \tilde{\gamma} \cot \tilde{\beta}\right.$ & 0 & 0 & $\sin a \chi \cos \tilde{\gamma} \csc \tilde{\beta}$ \\
\hline & & & & $\times \csc \chi / r_{2}$ & $-\cos a \chi] \csc \chi / r_{2}$ & & & $\times \csc \chi / i r_{2}$ \\
\hline \multirow[t]{2}{*}{0} & $\cos \bar{a} \chi$ & 0 & $-\sin \bar{a} \chi / r_{2}$ & $-a \sin \bar{a} \chi$ & $a \sin \bar{a} \chi \sin \tilde{\gamma} \cot \tilde{\beta} / r_{2}$ & 0 & 0 & $-a \sin \bar{a} \chi \sin \tilde{\gamma}$ \\
\hline & & & & $\times \cos \tilde{\gamma} / r_{2}$ & & & & $\times \csc \tilde{\beta} / i r_{2}$ \\
\hline 0 & 0 & 0 & 0 & $-1 / r_{3}$ & 0 & 0 & $1 / i r_{3}$ & 0 \\
\hline 0 & 0 & 0 & 0 & 0 & $\csc \tilde{\beta} / r_{3}$ & $-1 / i r_{3}$ & 0 & $-\cot \tilde{\beta} / i r_{3}$ \\
\hline 0 & 0 & 1 & 0 & 0 & 0 & 0 & 0 & 0 \\
\hline
\end{tabular}


TABLE IV: Coordinate transformation marrix $\mathbf{g}^{(4)}$.

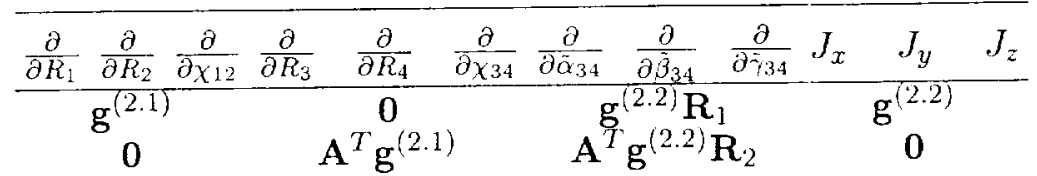

\begin{tabular}{|c|c|c|c|c|}
\hline \multirow{3}{*}{$\mathbf{R}_{1}$} & & $\cos \tilde{\alpha}_{34} \cot \tilde{\beta}_{34}$ & $\sin \tilde{\alpha}_{34}$ & $-\cos \tilde{\alpha}_{34} \csc \tilde{\beta}_{34}$ \\
\hline & $=$ & $\sin \tilde{\alpha}_{34} \cot \tilde{\beta}_{34}$ & $-\cos \tilde{\alpha}_{34}$ & $-\sin \tilde{\alpha}_{34} \csc \tilde{\beta}_{34}$ \\
\hline & & -1 & 0 & 0 \\
\hline \multirow{3}{*}{$\mathbf{R}_{2}$} & & $-\cos \tilde{\gamma}_{34} \csc \tilde{\beta}_{34}$ & $\sin \tilde{\gamma}_{34}$ & $\cos \tilde{\gamma}_{34} \cot \tilde{\beta}_{34}$ \\
\hline & $=$ & $\sin \tilde{\gamma}_{34} \csc \tilde{\beta}_{34}$ & $\cos \tilde{\gamma}_{34}$ & $-\sin \tilde{\gamma}_{34} \cot \tilde{\beta}$ \\
\hline & & 0 & 0 & 1 \\
\hline
\end{tabular}

$$
\begin{array}{cc}
\mathbf{A}^{T}=\mathbf{A}^{T}\left(\tilde{\alpha}_{34}, \tilde{\beta}_{34}, \tilde{\gamma}_{34}\right) & \mathbf{0} \\
\mathbf{0} & \mathbf{A}^{T}\left(\tilde{\alpha}_{34}, \tilde{\beta}_{34}, \tilde{\gamma}_{34}\right)
\end{array}
$$

